\title{
HRTEM Resolution Extension for Interface by Gerchberg-Saxton Algorithm with Supported Constraint
}

\author{
F.R. Chen*, U. Dahmen** and J. J. Kai* \\ * Dept. of Engineering and System Science, National Tsing Hua University, HsinChu, Taiwan, ROC \\ ** National Center for electron Microscopy-LBL, Berkeley, CA 94720, USA
}

When the electron beam passes through the thin specimen and objective lens, it forms diffraction pattern in back focal plane (reciprocal space) and high resolution image in the image plane (real space). Nevertheless, only partial information is preserved in either space. As in the case of $x$-ray diffraction, the modulus of the diffraction beams is recorded and the information of the phases lost in the back focal plane, although, it contains diffraction ampli tude up to $2 \AA^{-1}$. On the contrary, high resolution TEM (HRTEM) retains distorted phases in the low spatial frequency region (less than information limit) and the information in higher spatial frequency region were cut off by the lens contrast transfer function (CTF). For a medium high voltage TEM, the phase information contains in a HRTEM image may only up to about $0.5 \AA^{-1}$, so that direct correlation of the HRTEM image of crystal structure is not trivial. Gerchberg-Saxton algorithm has been utilized to reco ver the information from partial data in real and reciprocal spaces [ $\left[\begin{array}{ll}1 & -3\end{array}\right]$. The Gerchberg-Saxton algorithm restores spatial resolution by operating real space and reciprocal space projections cyclically. In our methodology, a generalized maximum entropy method (Kullback-Leibler cross entropy) dealing with weak object case is used as a real space (P1) projection. After P1 projection, not only the phases within the input spatial frequencies are improved, but also the phases in the higher frequencies are extrapolated. The optimum solutions from P1 projection can be further improved by a P2 projection that square root of diffraction intensities from a diffraction pattern are then substituted to complete a cycle operation of Gerchberg-Saxton algorithm. Sometimes, diffraction data may be incomplete or not available from irregular local defect such as dislocation or reconstructed interface which may make the performance of Gerchberg-Saxton algorithm harder, if not impossible. The same difficult exists in the case of surface direct method [4]. Marks has employed a prior knowledge called "support constraint" which was suggested in the image reconstruction literatures [5] to enhance the strength of the direct method for solving surface structure [4]. The concept of support constraint can be applied to the case of irregular local defect at the interface. A prior knowledge of known crystal structure in the perfect crystal region can be used as "supported constraint" by separating it from the local defect. New method has been developed to define quantitatively the support constraint region. The final process of Gerchberg-Saxton algorithm has to finish in the real space operator P1 in order to reveal the local defect at the interface. The detail procedures and mathematics will be discussed in detail in the talk. A HRTEM image of $\Sigma=5$ $\{210\}$ boundary at $\mathrm{TiO}_{2}$ is shown in FIG. 1(a). FIG. 1(b) is the final Gerchberg -Saxton solution of the square marked region in FIG. 1(a). The red and blue dots represent Ti and O, respectively. Example of a 2x1 interfacial reconstruction in the NiSi2/Si interface is shown in the FIG. 2(a). Super-periodicity of 2x1 necking contrast in FIG. 2(a) is exhibited. Without supplying the diffraction amplitude for the 2x1 interfacial reconstruction, the 2x1 super -periodicity can still be revealed. Gerchberg-Saxton solution of FIG. 2(a) is shown in FIG. 2(b). The necking contrast corresponds to deficient of Si column in the interface. 


\section{References}

[1] R. W. Gerchberg and W. O. Saxton, Optik 35 (1972) 709

[2] F.-R. Chen et al., J. of Electron Microscopy 48 (1999) 827-836

[3] F.-R. Chen et al., J. of Electron Microscopy (2002) in press

[4] L. D. Marks, Phys. Rev. B, 60 (1999) 2771

[5] J. C. Dainty and J. R. Fienup, (1987) Chap. 7, Restoration from phase and magnitude by generalized projections, In: Image Recovery: Theory and Application, ed. Stark H, pp231-275 (Academic Press, London)

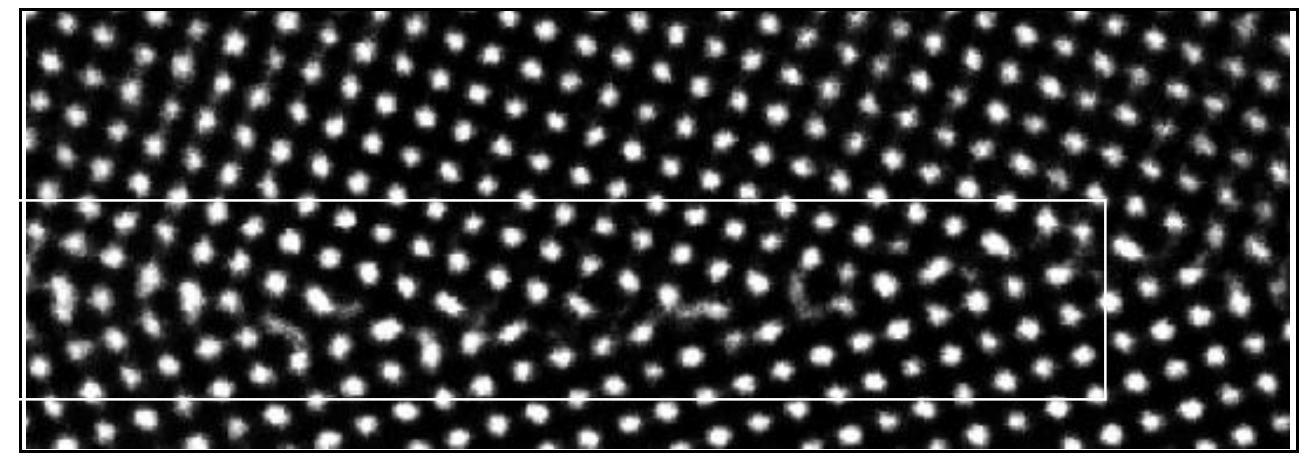

(a)

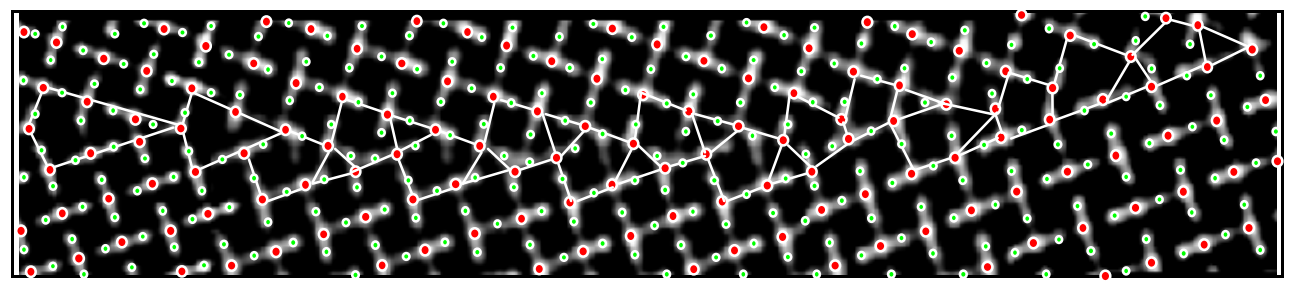

(b)

Fig. 1 a HRTEM image of $\Sigma=5\{210\}$ boundary in TiO2 (b) The Gerchberg-Saxton solution of (a). The red and blue dots represent $\mathrm{Ti}$ and $\mathrm{O}$, respectively.

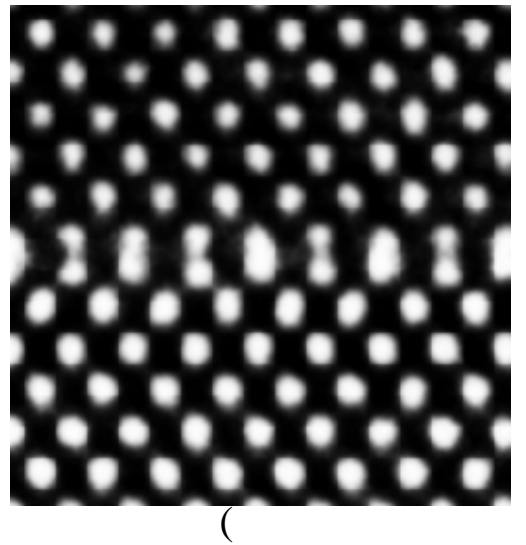

(a)

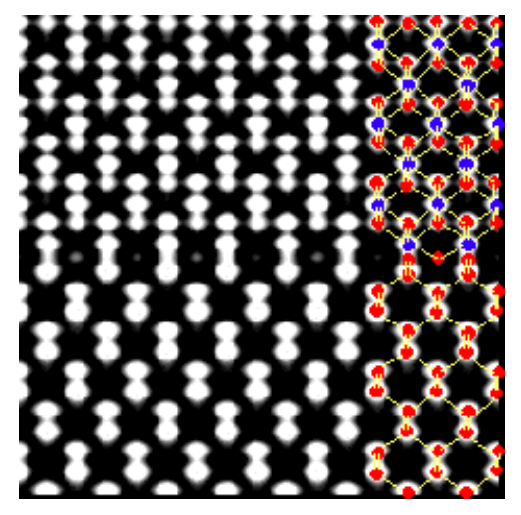

(b)

FIG. 2 (a) HRTEM image of type A NiSi $2 / \mathrm{Si}$ interface (b) The Gerchberg-Saxton solution for (a). The blue and red dots represent $\mathrm{Ni}$ and $\mathrm{Si}$, respectively. 